H. Boly ${ }^{1}$

A. Koubaye ${ }^{1}$

M.C. Viguier-Martinez ${ }^{2}$

A. Yenikoye ${ }^{3}$

\title{
Gestation et reprise de l'activité sexuelle après le part chez la brebis Djallonké, variété "Mossi"
}

BOLY (H.), KOUBAYE (A.), VIGUIER-MARTINEZ (M.C.), YENIKOYE (A.). Gestation et reprise de l'activité sexuelle après le part chez la brebis Djallonké variété "Mossi". Revue Élev. Méd. vét. Pays trop. 1993, 46 (4) : 631-636

Cette étude réalisée de juillet 1990 à avril 1991 vise à déterminer les caractéristiques physiologiques de la gestation et de la reprise de la cyclicité après le part chez la brebis Djallonké variété "Mossi”" $(\mathbf{n}=\mathbf{5 2})$. L'observation des gestations sur 20 brebis saillies naturellement en octobre et novembre (saison sèche et fraîche), permet d'évaluer à $149 \pm 2,4$ jours la durée de la gestation, à 92 p. 100 le taux de fertilité, à 118 p. 100 le taux de prolificité, à 108 p. 100 le taux de fécondité et à 7,1 p. 100 le taux de résorption embryonnaire. La croissance du foetus et de ses annexes est lente durant les 2 premiers mois $(<10 \mathrm{~g} / \mathbf{j})$. A partir du troisième mois, elle s'accélère avec $27 \mathrm{~g} / \mathrm{j}$. La progestéronémie plasmatique maternelle augmente et passe de $5,58 \pm 0,85 \mathrm{ng} / \mathrm{ml}$ à $13,71 \pm 2,32 \mathrm{ng} / \mathrm{ml}$ durant cette deuxième période. Sur 14 autres brebis, le suivi de l'activité sexuelle après la parturition, par observation de la reprise des chaleurs pour des mises bas survenues entre décembre et janvier (saison sèche et froide), indique III intervalle agnelage-premier nestrus de $56,6 \pm 10$ jours. Le suivi d'un groupe expérimental de 18 brebis placées en boxes individuels avec un niveau alimentaire variable $(80,100$ et 120 p. 100 des besoins) soumises à l'allaitement pendant 4,5 et 6 semaines montre que le délai d'apparition des chaleurs dépend à la fois de ce niveau alimentaire et de la durée de l'allaitement $(P<0,05)$. Une alimentation couvrant tous les besoins et un allaitement court de 4 semaines permettent d'avancer de près de trois semaines la reprise des chaleurs, soit 35 jours après l'agnelage. Le dosage de la progestérone plasmatique montre que les premières ovulations se produisent plus précocement, en moyenne $25 \pm 2$ jours après le part. En conclusion, une amélioration substantielle des performances de reproduction chez la brebis Djallonké variété "Mossi" est possible, à condition que l'apport alimentaire couvre les besoins et que le sevrage des agneaux soit précoce.

Mots clés : Ovin - Brebis - Mouton Djallonké - Activité sexuelle - Gestation - Performance de reproduction - Fertilité - Sevrage - Fœtus - Parturition - Cycle oestral - Progestérone - Allaitement - Alimentation des animaux - Burkina Faso

\section{INTRODUCTION}

La connaissance des caractéristiques de la gestation et de la reprise de l'activité sexuelle après le part est nécessaire à la mise en place d'une gestion rationnelle de la reproduction. Les données obtenues chez les races tropicales indiquent que ces parametres varient selon chaque

1. IDR, Université de Ouagadougou, 03 BP 7021, Burkina Faso. 2. Laboratoire de Neuro-endocrinologie, Faculté des Sciences, 37200 Tours, France.

3. Faculté des Sciences, Université de Niamey, Niger.

Reçu le 8.7.1992, accepté le 22.7.1993. race, les conditions climatiques et le mode d'élevage (2, $7,8,26)$. Quant aux mauvaises performances, elles sont liées à des avortements idiotypiques et à des reprises différées de la cyclicité $(1,5,9,10)$.

En vue du développement de l'élevage dans les zones soudano-sahéliennes par l'utilisation du mouton Djallonké (variété "Mossi") $(5,7,11,15)$, ces paramètres doivent être précisés en tenant compte de l'action spécifique des facteurs tels que la température, la photopériode, les carences alimentaires, le sevrage et la gestion du troupeau.

L'objet de cette étude est de préciser les caractéristiques de la gestation et de la reprise de l'activité cyclique de l'ovaire après le part chez cette brebis, en étudiant l'influence de l'alimentation et de la période de sevrage.

\section{MATÉRIEL ET MÉTHODES}

\section{Cadre expérimental et animaux}

Les brebis utilisées sont issues du troupeau de la station expérimentale de Gampela, située à une vingtaine de kilomètres à l'est de Ouagadougou ( $12^{\circ} 22$ latitude nord et $1^{\circ} 31$ longitude ouest). La ration alimentaire est composée de paille de sorgho haché (50 p. 100), de son de blé (20 p. 100), de tourteau de coton (15 p. 100), de drêche de brasserie (15 p. 100), de pierre à lécher et d'eau à volonté. Les conditions d'élevage ont été précédemment décrites (4).

L'étude s'est déroulée de juillet 1990 à avril 1991 et a consisté en deux essais : le 1er traitant de la gestation porte sur 20 brebis, le 2 e comprenant 32 brebis, concerne la reprise de la cyclicité après le part. Les brebis âgées de 2 à 6 ans ont été confirmées non gestantes et sans antécédents pathologiques de reproduction. Un bélier entier a été utilisé pour la saillie des femelles et un autre bélier vasectomisé muni d'un harnais marqueur a servi à la détection des brebis en chaleur.

Après 3 mois d'adaptation aux conditions expérimentales et de vérification de la régularité des cycles sexuels, les brebis ont subi le protocole expérimental décrit ci-après. 


\section{Protocole}

\section{Détermination des caractéristiques de la gestation (20 brebis)}

Une semaine après le jour de la lutte $=J_{0}$, une laparotomie exploratrice de l'état fonctionnel des ovaires est effectuée de façon à apprécier le nombre d'ovulations. La différence entre ce dernier et le nombre de produits obtenus permet d'avoir une estimation de la résorption embryonnaire (3). Les caractéristiques morphologiques du développement du foetus ainsi que celui de ses annexes sont suivies par hystérotomie effectuée à 1, 2, 3 et 4 mois de gestation sur 16 brebis, soit 4 brebis par mois. Le plasma sanguin de 4 brebis dont la gestation est suivie jusqu'au terme est recueilli quotidiennement pour le dosage radio-immunologique de la progestérone selon le protocole développé par YENIKOYE (29).

\section{Détermination de la reprise de l'activité sexuelle après le part (32 brebis)}

Elles sont répartis en deux lots :

- un lot témoin de 14 brebis soumis au système d'élevago semi-intensif de la station en présence permanente d'un bélier vasectomisé pour la détection des premières chaleurs. Les animaux sont en stabulation libre et reçoivent leur ration à partir d'une mangeoire et d'un abreuvoir collectifs. Les agnelages ont lieu de décembre à janvier (saison sèche et froide) et l'allaitement s'effectue sans intervention jusqu'au sevrage qui se produit naturellement lorsque les agneaux n'arrivent plus à couvrir leurs besoins et parviennent à ingérer la fraction ramollie de la ration alimentaire ;

- un lot expérimental de 18 brebis, issues de la même période d'agnelage que précédemment, réparties en 3 groupes de 6 brebis suivant une variation quantitative de leur ration de base : 80 p. 100 des besoins pour le groupe 1,100 p. 100 pour le groupe 2 et 120 p. 100 pour le groupe 3. Chaque groupe de niveau alimentaire ainsi défini est à son tour subdivisé en 3 sous-groupes correspondant à des périodes de sevrage de 4,5 et 6 semaines (tabl. I). La progestérone plasmatique des 2 brebis du groupe 3 sevrées à 4 semaines, a été dosée selon le même protocole (28).

TABLEAU I Répartition des brebis selon le niveau alimentaire et la durée de l'allaitement.

\begin{tabular}{|c|c|c|c|c|c|c|c|c|}
\hline \multirow{3}{*}{$\begin{array}{l}\text { Niveau alimentaire } \\
\text { Allaitement } \\
\text { (durée en semaine) } \\
\text { Nombre de brebis }\end{array}$} & \multicolumn{2}{|c|}{$\begin{array}{l}\text { Groupe } 1 \\
(80 \text { p. } 100)\end{array}$} & \multicolumn{3}{|c|}{$\begin{array}{c}\text { Groupe } 2 \\
(100 \text { p. } 100)\end{array}$} & \multicolumn{3}{|c|}{$\begin{array}{l}\text { Groupe 3) } \\
(120 \text { p. } 100\end{array}$} \\
\hline & & 56 & 4 & & 6 & 4 & 5 & 6 \\
\hline & 22 & 22 & 2 & 2 & 2 & 2 & 2 & 2 \\
\hline
\end{tabular}

\section{Définition des paramètres de reproduction}

Le taux de fertilité est défini comme étant le nombre de brebis pleines par rapport aux brebis mises à la reproduction. Le taux de prolificité est le nombre d'agneaux nés par brebis mettant bas et le taux de fécondité représente le nombre d'agneau nés par femelle mise à la reproduction.

\section{Analyse statistique}

Les résultats obtenus ont été analysés au moyen du système SAS (25). L'étude des relations entre les différentes variables a été effectuée par les tests de corrélation de Pearson. Les effets des différents facteurs de variation sur la reprise de la cyclicité après le part ont été appréciés par l'analyse de variance non orthogonale à effet fixe (Proc.GLM). Les résultats sont exprimés en moyenne \pm écart-type et les différences considérées comme significatives au seuil de probabilité de $\mathrm{P}<0,05$.

\section{RÉSULTATS}

\section{Caractéristiques de la gestation}

\section{Développement du fœtus et ses annexes embryonnaires - Durée de la gestation}

Au premier mois, il est impossible de faire des mensurations précises sur le foetus. A partir du 2e mois, les différentes configurations morphologiques deviennent perceptibles. L'évolution des mensurations du foetus et des enveloppes est rapportée dans le tableau II. La croissance est lente durant les deux premiers mois $(<10 \mathrm{~g} / \mathrm{j})$; elle s'accélère à partir du $3 e$ (environ $27 \mathrm{~g} / \mathrm{j})$. La longueur totale ou atlato-ischiale $(r=0,99 \mathrm{P}<0,005)$, la hauteur au garrot $(r=0,99 P<0,01)$ et le volume des enveloppes foetales $(r=0,99 \mathrm{P}<0,05)$ sont étroitement corrélés à l'âge du foetus. La durée moyenne de la gestation est de $149,8 \pm 2,4$ jours.

\section{Profil de la concentration de la progestérone plasmatique}

La concentration moyenne de la progestérone pendant la gestation est de $9,64 \pm 1,13 \mathrm{ng} / \mathrm{ml}$. Son évolution comprend cependant deux phases : l'une avec une concentration basse de $5,58 \pm 0,85 \mathrm{ng} / \mathrm{ml}$ durant $82,5 \pm 1,4$ jours et l'autre de niveau plus élevé : $13,71 \pm 2,32 \mathrm{ng} / \mathrm{ml}$ qui se maintient jusqu'au terme de la gestation. Les niveaux maximaux sont obtenus entre le $116 \mathrm{e}$ et le $126 \mathrm{e}$ jour (fig. 1). 
TABLEAU II Mensuration du fotus et des annexes fotales (moyenne \pm écart-type).

\begin{tabular}{|c|c|c|c|c|c|c|c|c|c|}
\hline $\begin{array}{c}\text { Age } \\
\text { (mois) }\end{array}$ & $\begin{array}{l}\text { PF } \\
\text { (g) }\end{array}$ & $\begin{array}{c}\text { GMQ } \\
(\mathrm{g})\end{array}$ & $\begin{array}{l}\text { PEF } \\
\text { (g) }\end{array}$ & $\begin{array}{l}\text { VF } \\
(\mathrm{ml})\end{array}$ & $\begin{array}{l}\text { VEF } \\
(\mathrm{ml})\end{array}$ & $\begin{array}{l}\mathrm{LA} \\
(\mathrm{cm})\end{array}$ & $\begin{array}{l}\mathrm{TT} \\
(\mathrm{cm})\end{array}$ & $\begin{array}{l}\mathrm{HG} \\
(\mathrm{cm})\end{array}$ & Observations \\
\hline 1 & - & & - & - & - & - & - & - & \\
\hline 2 & $9,1 \pm 0,8$ & $0,152 \pm 0,04$ & $18 \pm 0,05$ & $150 \pm 2,2$ & $200 \pm 1,8$ & $5,6 \pm 0,02$ & $5,08 \pm 0,04$ & $3,26 \pm 0,01$ & $\begin{array}{l}\text { Début mouvements } \\
\text { respiratoires }\end{array}$ \\
\hline 3 & $319 \pm 1,8$ & $\begin{array}{l}9,96 \pm 0,1 \\
26,7 \pm 0,15\end{array}$ & $350 \pm 0,77$ & $305 \pm 1,8$ & $396 \pm 1,4$ & $16 \pm 0,55$ & $13 \pm 0,60$ & $11+0,12$ & $\begin{array}{l}\text { Ouverture } \\
\text { des lèvres }\end{array}$ \\
\hline 4 & $1090 \pm 0,4$ & $28,7 \pm 0,18$ & $566 \pm 0,11$ & $850 \pm 4,0$ & $620 \perp 0,8$ & $30 \pm 0,7$ & $29 \pm 0,8$ & $26 \pm 0,3$ & $\begin{array}{l}\text { Mise en place } \\
\text { des phanères }\end{array}$ \\
\hline 5 & $1950 \pm 0,35$ & & $610 \pm 0,08$ & 一 & - & $40 \pm 1,2$ & $33,5 \pm 0,9$ & $38,5 \pm 0,72$ & \\
\hline
\end{tabular}

PF : poids fotal: GMQ : gain moyen quotidien; PEF : poids des enveloppes fotales; VF : volume foetal; VEF : volume des enveloppes fœtales ; LA : longueur atlato-ischiale; TT: tour du thorax; HG: hauteur au garrot.

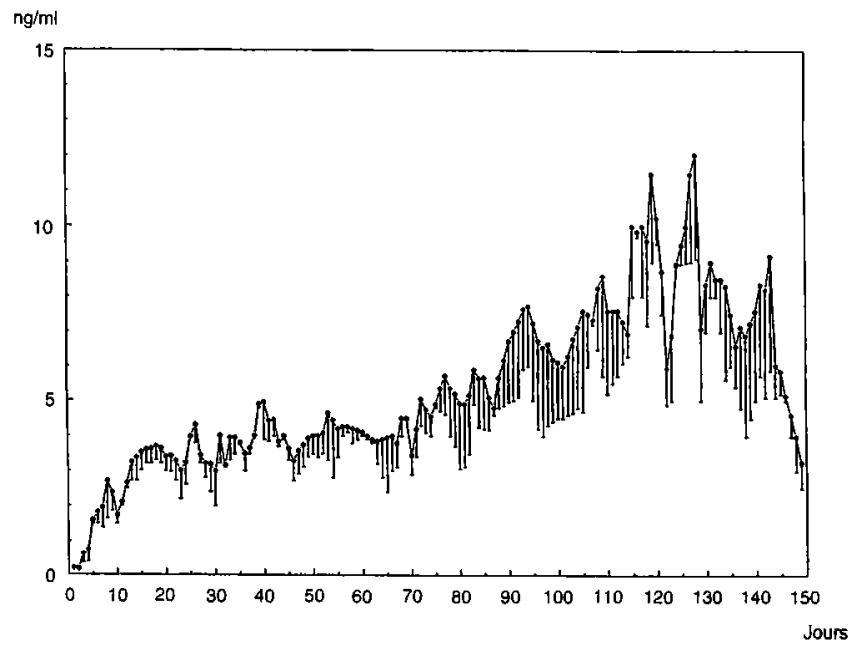

Figure I: Progesteronémie au cours de la gestation (Moy. \pm SEM). (SEM : Somme des écarts par rapport à la moyenne)

\section{Les paramètres de reproduction}

En moyenne 1,16 corps jaune par brebis avec 75 p. 100 des corps jaunes répartis sur l'ovaire droit ont été observés après laparotomie à $\mathrm{J}_{7}$. Sur l'ensemble des brebis observées, les taux de fertilité, prolificité, fécondité et résorption embryonnaire sont respectivement de 92, 118, 108 et 7,1 p. 100.

\section{Reprise de la cyclicité ovarienne}

\section{Durée}

L'intervalle moyen entre l'agnelage et la première chaleur est de $56 \pm 10$ jours. Le dosage de la progestérone plasmatique chez les deux brebis du groupe 3 indique cependant une reprise d'activité ovarienne plus précoce à
$24,5 \pm 2$ jours (fig. 2). Ces premières ovulations ne sont pas suivies de signes de chaleur qui apparaissent au cours de la $2 e$ ovulation.

\section{Influence de l'alimentation}

Les 12 brebis recevant 100 et 120 p. 100 des besoins nutritionnels ont été observées pour la première fois en chaleur, après le part, en moyenne 48,8 $\pm 5,0$ et $42,2 \pm 4,0$ jours, respectivement $(P>0,05)$. Ces intervalles sont significativement inférieurs $(P<0,05)$ à celui du groupe recevant 80 p. 100 de ses besoins nutritionnels avec $60,8 \pm 6,5$ jours.

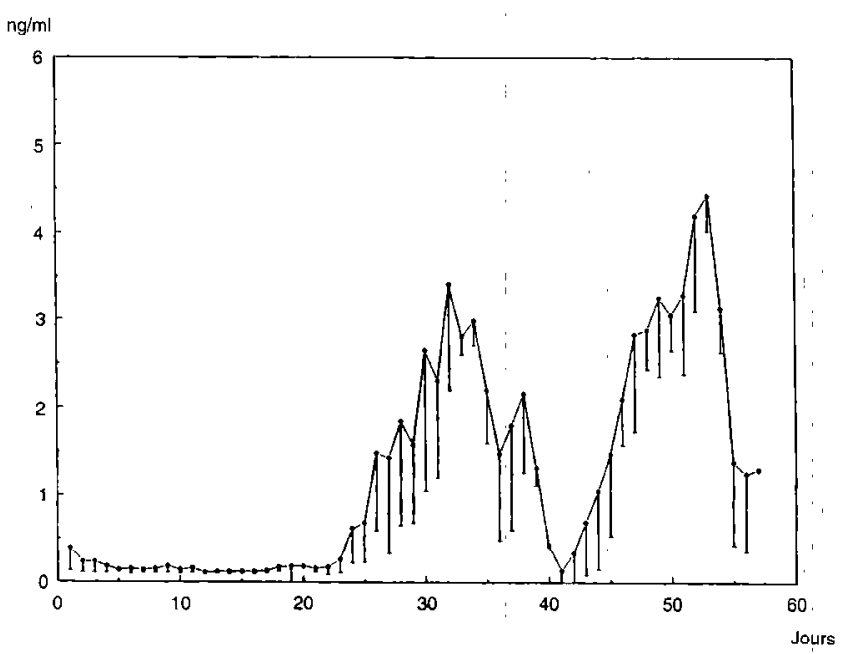

Figure 2 : Progesteronémie au cours de la reprise de la cyclicité (Moy. \pm $S E M)$. 


\section{L'influence de la période d'allaitement}

L'allaitement durant 5 et 6 semaines entraîne une reprise des chaleurs, respectivement à $54,5 \pm 5,2$ et $56,6 \pm 4,0$ jours, délai significativement plus long que dans le cas d'un allaitement de 4 semaines où le retour des chaleurs a lieu à $43,3 \pm 6$ jours après le part $(P<0,05)$. Ce facteur est donc important pour la conduite de l'élevage.

\section{Influence combinée du niveau alimentaire et de la période de sevrage}

En associant l'allaitement de 4 semaines aux niveaux alimentaires couvrant 100 et 120 p. 100 des besoins, on obtient la reprise précoce des chaleurs à $34,5 \pm 1,5$ jours. Lorsque le régime alimentaire couvre seulement 80 p. 100 des besoins, la diminution de la période d'allaitement ne permet pas une réduction significative $(P>0,05)$ de l'anoestrus post-partum (fig. 3 ).

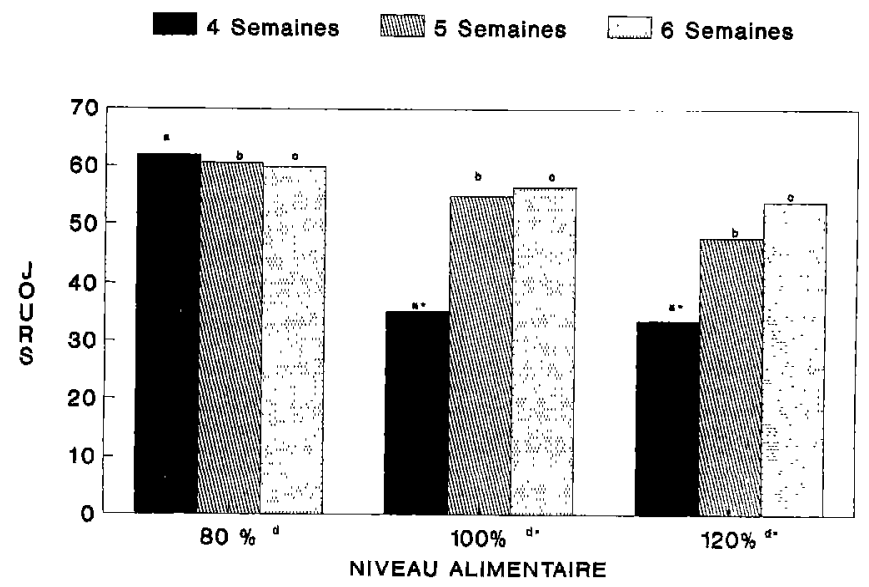

Figure 3 : Effets du niveau alimentaire et de la durée de l'allaitement (*indique la différence significative à $P<0,05$ ).

\section{DISCUSSION}

Avec $149 \pm 2$ jours, la durée de la gestation de la brebis Djallonké variété Mossi est comparable à celle d'autres races tropicales et européennes : 149 à 152 pour la brebis Peul du Niger (30), 148,5 jours pour la brebis Djallonké de Côte-d'Ivoire (16) et 149,3 jours pour les brebis Mérinos $x$ Dorset (24). Au cours de la gestation, le profil de la progestéronémie présente une première phase de sécrétion basse $(5,58 \pm 0,85 \mathrm{ng} / \mathrm{ml})$, plus longue $(82,5$ jours) comparativement au 70 jours de la brebis Peulh du Niger (29). A partir du 3e mois, il se produit une augmentation de la progestérone oscillant entre 12,07 et $15,36 \mathrm{ng} / \mathrm{ml}$. Ces valeurs sont supérieures à celles observées chez la brebis Peul (29), ce qui pourrait être lié au. taux de prolificité plus élevé chez la brebis Djallonké variété Mossi.
Les ratios de reproduction sont caractérisés par un taux de prolificité (118 p. 100) semblable à celui de la race Djallonké du Cameroun : 117 p. 100 (24) mais', en revanche, supérieur à ceux des races Djallonké de Côted'Ivoire : 112 p. 100 (2) et Peul du Niger : 106 p. 100 (29). Le taux de fécondité (108 p. 100) est inférieur à celui des brebis Djallonké de Côte-d'Ivoire : 149 p. 100 (2) ou Djallonké du Cameroun : 168 p. 100 (27). Cela pourrait être lié à l'origine de cette variété de Djallonké "Mossi" qui est le produit du croisement entre les Djallonké de la zone soudano-guinéenne avec fort taux de fécondité $(2,27)$ et les races Peul du Sahel moins fécondes (29). Le taux de résorption embryonnaire de 7,1 p. 100 est également inférieur à ceux des races maghrébines : 15 à 35 p. $100(20)$ et européennes : 25 p. 100 (3). Ce faible taux peut être lié au faible taux d'ovulation (22), au faible rythme de croissance pendant les premières phases de développement embryonnaire ou tout simplement le résultat d'une alimentation satisfaisante dans un environnement sanitaire bien maîtrisé. Ce résultat mérite cependant d'être confirmé par l'utilisation des techniques écographiques qui permettent des observations plus précoces et un suivi continu sur un plus grand nombre de brebis.

Le délai de la reprise de l'activité sexuelle de la brebis Djallonké variété Mossi par observation des chaleurs sur 14 brebis maintenues en élevage semi-intensif est proche de celui d'autres brebis comme la Djallonké de Côte-d'Ivoire et la brebis Oudah qui ont respectivement 60 (16) et 61 jours (12). En revanche, elle est tardive comparativement aux 42 jours de la brebis Peulh du Niger élevée en station (28). Cette valeur est proche des résultats de cette étude en box avec alimentation et sevrage contrôlés et confirme l'influence des effets troupeau et mode de suivi des reproducteurs (19). Lorsque la taille du troupeau est réduite, il y a très peu de compétition alimentaire entre les animaux. Le dosage de la progestérone indique que l'activité cyclique de l'ovaire reprend 3 à 4 semaines après le part. Les chaleurs apparaissent surtout au cours du 2 e cycle ovulatoire et ceci confirme d'autres observations faites chez les ovins (23), et les bovins $(17,21)$. La réduction du délai de la reprise des chaleurs après le part paraît dépendre surtout du niveau alimentaire. Pour un sevrage à 4 semaines, une alimentation couvrant les besoins de l'animal (100 p. 100 et 120 p. 100) permet d'avancer la reprise des chaleurs de 22 jours par rapport au lot alimenté à 80 p. 100 des besoins. La sous-alimentation, tout comme l'allaitement, exerce des effets inhibiteurs sur la décharge ovulatoire de $\operatorname{LH}(6,13,18)$ et induit une baisse de la sensibilité ovarienne aux gonadotropines $(14,17)$. La mise en place d'un protocole associant la détection des premières chaleurs avec un suivi endocrinien (LH et progestérone) devrait permettre de préciser si l'effet négatif de la sousalimentation s'exerce sur la reprise de l'activité cyclique de l'ovaire ou sur l'expression des chaleurs lors des premières ovulations. 


\section{CONCLUSION}

La brebis Djallonké variété Mossi possède une durée de gestation de $149,8 \pm 2,4$ jours. Les ratios de reproduction sont les suivants : fertilité : 92 p. 100, fécondité : 108 p. 100 , prolificité : 118 p. 100 et résorption embryonnaire : 7,1 p. 100 . L'étude montre que la reprise des chaleurs après le part est de l'ordre de 2 mois, mais cet intervalle est soumis à des variations liées à l'alimentation et la durée de l'allaitement. Un régime couvrant les besoins de la mère, associé à un sevrage précoce à 4 semaines des agneaux, permettent une réduction de près de moitié du délai de la reprise des chaleurs. Ceci contribue à améliorer significativement les performances de reproduction de la brebis Djallonké variété Mossi, du moins dans des conditions d'élevage bien contrôlées.

\section{REMERCIEMENTS}

Les auteurs adressent leurs remerciements au Dr M. THIBIER, Directeur du Laboratoire pour le Contrôle des reproducteurs (UNCEIA) à Maisons-Alfort (France) et au Dr J.C. MARIANA, de la Station de Physiologie de la reproduction (INRA, Nouzilly, France) pour leurs conseils éclairés.

\section{BIBLIOGRAPHIE}

1. AMEGEE (Y.). La prolificité du mouton Djallonké en milieu villageois au Togo. Revue Elev. Méd. vét. Pays trop., 1983, 36 (1) : 85-90.

2. BERGER (Y.), GINISTY (L.). Bilan de 4 années d'étude de la race ovine Djallonké en Côte-d'Ivoire. Revue Élev. Méd. vét. Pays trop., 1980 , 33 (1) : 71-78.

3. BERTRAND (M.). Mortalité embryonnaire. Inf. tech. Serv. vét., 1972. $39-40: 25-27$.

4. BOLY (H.), MAGAGI (L.), KONATE (T.), VIGUIER-MARTINEZ (M.C.), YENIKOYE (A.). Cycle ostral et croissance folliculaire de la brebis Djallonké variété "Mossi". Revue Elev. Méd. vét. Pays trop., 1992. $45(3-4): 335-340$

5. BOURZAT (D), BONKOUNGOU (E), RICHARD (D), SANFO (R.) Essais d'intensification de la production animale en zone sahélo-soudanienne : alimentation intensive des jeunes ovins dans le Nord du Burkina. Revue Élev. Méd. vét. Pays trop., 1987, 40 (2) : 151-156.

6. CARRUTHERS (T.D.), HAFS (H.D.). Suckling and four times daily milking, influence on ovulation oestrus and serum-luteinizing hormone glucocorticoid and prolactin in post-partum Holsteins. J. Anim. Sci.. I980, $50: 919-925$.

7. CHARRAY (J.), HAVET (A.). Performance de reproduction de brebis naines de l'Afrique de l'Ouest entretenues en région centre de la Côted'Ivoire. In : Colloque sur la reproduction des ruminants en zone tropicale. Petit-Bourg (Guadeloupe, France), 8-10 juin $1983.10 \mathrm{p}$.

8. CHEMINEAU (P.), MALPAUX (B.), PELLETIER (J.), DELGADILLO (J.A.), GUERIN (Y.), THIMONIER (J.). Effets de la lumière et de la tern- pérature sur la production des petits ruminants. In : Journée de l'Association pour l'étude de la reproduction, Maisons-Alfort, 25 janvier 1990 . F111.

9. DIANDA (D.). Contribution à l'étude des paramètres zootechniques de l'élevage traditionnel ovin dans le bloc pastoral de Sondré-Est. Mémoire fin d'étude. Ouagadougou, IDR, $1981.70 \mathrm{p}$.

10. DIARRA (D.). Étude des paramètres de la reproduction des ovins de Gampela. Mémoire fin d'étude. Ouagadougou, IDR, 1989.67 p.

11. JUMAS (R.), RAYMOND (H.). L'élevage des petits ruminants dans les circonscriptions de Kaya, Ouahigouya et du Sahel. Paris, SEDES, 1974.

12. GAILLARD (Y.). Caractéristiques de la reproduction de la brebis oudah. Revue Élev. Méd. vét. Pays trop., 1979, 32 (3) : 285-290.

13. GAUTHIER (D.), YAOUANC (A.), COCHAUD (J.), MAULEON (P.). Influence d'une sous-alimentation de la vache allaitante sur l'induction de l'ovulation par l'hormone gonadotrope sérique (PMSG) au cours du post-partum. Reprod. Nutr. Dév., 1981, 21 : 577-583.

14. GINISTY (L.). Amélioration de la productivilé des petits runinants. Rapport annuel 1977. Bouaké, Côte-d’Ivoire, CRZ, 1978.

15. GOURO (A.S.). Contribution à l'étude de l'activité ovarienne de la vache zébu Azawak par le dosage radio-immunologique de la progestérone plasmatique. Bull. Dossiers tech. vét. GTV, 1988.

16. HAUMESSER (J.B.), GREBALDI (P.). Observation sur la reproduc tion et l'élevage du mouton Oudah nigérien. Revue Élev. Méd. vét. Pays trop., 1980, 33 (2) : 205-213.

17. HUMBLOT (P.). Les paramètres de l'anoestrus 'post-partum. In Compte-rendu des journées de l'information UNCEIA-ITEB, mars 1982. Paris, ITEB, $1982:$ 5-18.

18. HUMBLOT (P.), THIBIER (M.). Anomalies fonctionnelles de là reproduction chez la vache. $I n$ : Journée d'information UNCEIA-ITEB novembre 1977. Paris, ITEB, 1978. p. 66-88.

19. HUMBLOT (P.), THIBIER (M.). Utilisation de schémas thérapeutiques dans un programme de contrôle de l'infécondité dans un troupeau dé vaches laitières. Recl. Méd. Vét., 1981, 157:91-104.

20. LAHLOU-KASSI (A.). Reproduction ovine. Maroc, Rabat, Institut agronomique et vétérinaire (IAV) Hassan II, 1991. 54 p.

21. MORROW (D.A.), ROBERTS (S.J.), McENTREE (H.), GRAY (H.G.). Post-partum ovarian activity and uterine involution in dairy cattle J. Am. vet. med. Ass., 1966, 149 : 1596-1609.

22. RICORDEAU (G.). POIVEY (J.P.), LAJOUS (D.), EYCHENNE (F.) Genetic aspects of ovulation rate and embryo mortality in Romanov ewes. In : DICKERSON (G.E.). JOHNSON (R.K.) Eds. Proceedings of the 3rd World congress on genetics applied to livestock production, Lincoln, Nebraska, USA. July 16-22. 1986. XI. Genetics of reproduction, lactation, growth, adaptation disease, and parasite resistance. The University of Nebraska Board of Regents. 1986. P. 90-95.

23. SHIRAR (A.), COGNIE (Y.), LOUALLT (F.). LEVASSEUR (M.C.) MARTINET (J.). Resumption of oestrous behaviour and cyclic ovarian activity in suckling and non suckling ewes. J. Reprod. Fert., 1989, 87 789-794.

24. SMITH (M.F.). Hormon treatment and use of calf removal in post-partum beef cows. I. Anim. Sci., 1987, 48 : 1285-1294.

25. Statistical Analysis System, SAS Institute Inc. SAS/STAT Guide for personal computers, version 6. Cary, USA, SAS, 1987 
26. THIMONIER (J.), TERQUI (M.), CHEMINEAU (P.). Conduite de la reproduction des petits ruminants dans les différentes parties du monde. In : Nuclear and related techniques in Animal production and health. Proceedings of a symposium, Vienna, 17-21 March 1986, jointly organized by IAEA and FAO. Vienna, IAEA, 1986. P. 135-147.

27. VALLERAND (F.), BRANCKAERT (R.). La race ovine Djallonké au Cameroun : potentialités zootechniques, condition d'élevage, avenir. Revue Élev. Méd. vét. Fays trop., 1975, 28 (4) : 523-545.

28. YENIKOYE (A.). Variations annuelles du comportement d'œstrus du taux et des possibilités d'ovulation chez la brebis Peulh du Niger. Reprod. Nutr. Dév., 1984, 24 (1) : 11-19.

BOLY (H.), KOUBAYE (A.), VIGUIER-MARTINEZ (M.C.), YENIKOYE (A.). Pregnancy and resumption of sexual activity after parturition in "Mossi" Djallonké ewes. Revue Élev. Méd. vét. Pays trop., $1993,46(4): 631-636$

The present study (July 1990-April 1991) aimed at determining some physiological parameters of pregnancy and resumption of cyclicity after parturition in Djallonké "Mossi" ewes ( $n=52$ ). The observation of 20 ewes naturally mated between October and November (dry and cool season) showed a pregnancy length of $149 \pm 2.4$ days, a fertility rate of $92 \%$, a prolificacy rate of $118 \%$, a fecundity rate of 108 and $7.1 \%$ of embryoresorption. Foetal growth and that of its membranes were slow $(<10 \mathrm{~g} / \mathrm{day})$ during the first two months. After three months, the growth rate increased (about $27 \mathrm{~g} /$ day). The maternal plasma progesterone concentration rose during this period from $5.58 \pm 0.85$ to $13.71 \pm 2.32 \mathrm{ng} / \mathrm{ml}$. The study of cyclicity resumption (December-January) conducted in 14 other ewes showed that the first oestrus appeared 56.6 \pm 10 days after parturition. A trial made in 18 cwes housed in individual boxes and receiving different levels of feeding $(80,100$ and $120 \%)$, and with a suckling period of 4,5 and 6 weeks showed that the return to heats was related both to the level of feeding and to the suckling length. A dietary regimen meeting all nutrient requirements and a suckling period of 4 weeks led to reducing the interval from parturition to first oestrus by about 3 weeks, i.e. 35 days after lamhing. The maternal plasma progesterone levels showed that the first ovulation occured $25 \pm 2$ days after parturition. In conclusion, these parameters indicate that the reproductive performances of Mossi Djallonké ewies may be improved provided that the feed supply is large enough and that the lambs are weaned early.

Key words : Ewe - Djallonké sheep - Sexual activity - Pregnancy - Reproductive performance - Fertility - Weaning - Foetus - Lambing - Oestrous cycle - Progesterone - Suckling - Animal feeding - Burkina Faso.
29. YENIKOYE (A.). Étude de l'endocrinologie sexuelle et de la croissance folliculaire chez la brebis nigérienne de race Peulh : influence de la sáison de reproduction. Thèse doct. es-Sci. Nat. Tours, Université François Rabelais, 1986. p. 96.

30. YENIKOYE (A.). Variations saisonnières des teneurs plasmaticues de PRL, FSH et LH et du rétrocontrôle de FSH et LH chez la brebis Peulh du Niger. In :African small ruminant research and development : Proceedings of a conference held at Bamenda, Cameroun, 18-25 January 1989. Addis Ababa, Ethiopie, Africa small ruminant research network, ILCA, 1989. P. $287-302$.

BOLY (H.), KOUBAYE (A.), VIGUIER-MARTINEZ (M.C.), YENIKOYE (A.). Gestación e inicio de la actividad sexual post parto, en la oveja Djallonké, variedad "Mossi". Revue Elev. Méd. vét. Pays trop., 1993, $46(4):$ 631-636

El estudio se realizó entre julio 1990 y abril 1991. El objetivo fue determinar las características fisiológicas de la gestación y del inicio de la ciclicidad post parto en la oveja Djallonklé, variedad "Mossi" ( $=52$ ). La observación de los períodos de gestación de 20 ovejas, expuestas a monta natural entre octubre y noviembre (estación seca y fresca), permitió la determinación de la duración de la gestación $(149 \pm 2,4$ días) de la tasa de fertilidad $(92$ p. 100), de la tasa de prolificidad (118 p. 100), de la tasa de fecundidad (108 p. 100) y de la tasa de reabsorción embrionaria $(7,1$ p. 100). El crecimiento del feto y de sus anexos es lento durante los dos primeros meses $(<10$ g/día), aumentando a partir del tercer mes ( 27 g/día). Durante este úllimo período, la progesterona plasmática materna aumenta, pasando de $5,58 \pm 0,85 \mathrm{ng} / \mathrm{ml}$ a $13,71 \pm 2,32 \mathrm{ng} / \mathrm{ml}$. Se siguió la actividad sexual post parto de otras 14 ovejas, mediante la observación de retorno a celo en animales que parieron entre diciembre y enero (estación seca y fría), obteniéndose un intervalo parto-primer celo de $56,6 \pm 10$ días. La observación experimental de 18 ovcjas en cstabulación individual, con un nivel alimenticio variable $(80,100$ y 120 p. 100 de las necesidades) y amamantando durante períodos de 4,5 y 6 semanas, muestra que el retraso en la aparición de celos depende, tanto del nivel alimenticio, como del momento del destete $\left(P^{\prime}<0,05\right)$. Una alimentación que satisfaga todas las necesidades y un período de amamantamiento corto, de 4 semanas, permiten un retorno a celo tres semanas antes, es decir, 35 días post-parto. La dosificación de la progesterona plasmática muestra que las primeras ovulaciones se producen antes, alrededor de $25 \pm 2$ días post parto. Se concluye que una mejora substancial de los índices reproductivos en la oveja Djallonké variedad Mossi es posible, siempre y cuando el aporte nutricional cubra las necesidades y el destete de los corderos sea precoz.

Palabras claves: Oveja - Ovino Djallonké - Actividad sexual - Gestación - Reproductividad - Fertilidad - Destete - Feto - Parto - Ciclo estral - Progesterona - Lactancia - Alimentación animal - Burkina Faso. 